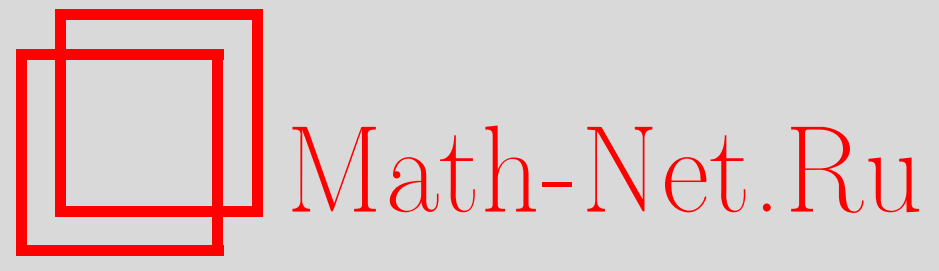

В. П. Воронин, О средних и типичных значениях сумм попарных расстояний для подмножеств вершин $n$-мерного единичного куба, Дискрет. матем., 2004, том 16, выпуск $3,141-152$

DOI: https://doi.org/10.4213/dm168

Использование Общероссийского математического портала Math-Net.Ru подразумевает, что вы прочитали и согласны с пользовательским соглашением http://www . mathnet.ru/rus/agreement

Параметры загрузки:

IP : 54.198 .64 .247

26 апреля 2023 г., $17: 23: 52$

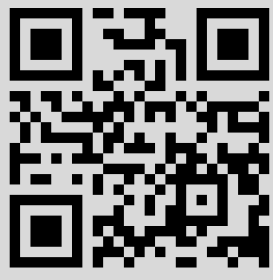




\title{
Аискретная математика
}

том 16 выпуск $3 * 2004$

УдК 519.6

\section{О средних и типичных значениях \\ сумм попарных расстояний \\ для подмножеств вершин \\ n-мерного единичного куба}

\author{
๑) 2004 г. В. П. Воронин
}

В работе исследуется вопрос о средних и типичных значениях сумм попарных расстояний Хэмминга для подмножеств вершин $n$-мерного единичного куба. Описан подход к проблеме вычисления средних и типичных значений для произвольных функционалов, определенных на подмножествах конечного множества как сумма значений, приписываемых упорядоченным парам элементов этого множества, получены общие формулы для этого случая. Найдены средние и типичные значения сумм попарных расстояний для случая всех подмножеств вершин $n$-мерного единичного куба и сумм попарных расстояний для подмножеств вершин фиксированной мощности.

Работа выполнена при поддержке Российского фонда фундаментальных исследований, проект 01-01-00266а.

\section{1. Введение}

Пусть $B^{n}-$ множество вершин $\tilde{\alpha}^{n} n$-мерного единичного куба, $\rho\left(\tilde{\alpha}^{n}, \tilde{\beta}^{n}\right)-$ расстояние Хэмминга между вершинами $\tilde{\alpha}^{n}$ и $\tilde{\beta}^{n}$. Пусть

$$
M=\left\{\tilde{\alpha}_{1}^{n}, \tilde{\alpha}_{2}^{n}, \ldots, \tilde{\alpha}_{m}^{n}\right\}
$$

- $m$-элементное подмножество вершин $B^{n}$. Представляет интерес изучение функционалов вида

$$
F(M)=\sum_{1 \leqslant i<j \leqslant m} \varphi\left(\rho\left(\tilde{\alpha}_{i}^{n}, \tilde{\alpha}_{j}^{n}\right)\right)
$$

где $\varphi(n)$ - некоторая функция, определенная на натуральных числах.

С. В. Яблонским была поставлена задача нахождения $m$-элементного подмножества вершин $B^{n}$, которое доставляет минимум такому функционалу для случая

$$
\varphi(n)=\frac{1}{n}
$$

Этот функционал называется энергией множества одноименно заряженных частиц. В [1] эта проблема была решена для случая $m=2^{n-1}$. В [2] была рассмотрена асимптотическая постановка этой проблемы. В. К. Леонтьевым в [3] была найдена асимптотика 
для функщионала энергии в случае произвольной растущей мощности подмножеств. Показано, что почти все $m$-подмножества имеют асимптотически минимальную энергию и найдена оценка отклонения для почти всех множеств. Для достаточно быстро растущей мощности подмножеств (как функции размерности куба) построены подмножества с асимптотически минимальной энергией. Была попытка исследовать подмножества с максимальной энергией [4], но описать достаточно точно структуру этих подмножеств и получить оценки значений не удалось.

В настоящей работе рассматривается случай

$$
\varphi(n)=n,
$$

то есть функционал суммы попарных расстояний между вершинами подмножества.

\section{2. Вспомогательные утверждения}

В этом разделе описывается общий подход к задаче вычисления средних и типичных значений для случая функционалов, определенных на декартовых квадратах конечного множества. Выводятся некоторые вспомогательные формулы, необходимые для вычисления средних значений и оценок для типичных значений суммы попарных расстояний. Техника вычислений математических ожиданий и дисперсий, предложенная В. К. Леонтьевым в [3], используется и обобщается в настоящей работе на произвольные квадратные матрицы.

Пусть дано $r$-элементное множество. Пусть это будет $I=\{1,2, \ldots, r\}$, что не ограничивает общности. Пусть имеется некоторая функция $\varphi$, определенная на декартовом квадрате $I^{2}$ и сопоставляющая каждой упорядоченной паре $(i, j)$ действительное число $a_{i j}$. Эту функцию можно представить в виде квадратной матрицы $A$ порядка $r$, элементы которой соответствуют значениям функции $\varphi$,

$$
A=\left(\begin{array}{cccc}
a_{11} & a_{12} & \ldots & a_{1 r} \\
a_{21} & a_{22} & \ldots & a_{2 r} \\
\ldots \ldots & \ldots & \ldots & \ldots \\
a_{r 1} & a_{r 2} & \ldots & a_{r r}
\end{array}\right)
$$

На пересечении $i$-й строки и $j$-го столбца находится элемент $a_{i j}$ - значение функции $\varphi$ на паре $(i, j)$.

Функщионал $F$, определенный на подмножествах множества $I$, задается следующим образом. Пусть $M \subseteq I$, тогда

$$
F(M)=\sum_{i \in M, j \in M} \varphi(i, j)=\sum_{i \in M, j \in M} a_{i j}
$$

то есть, если задано множество $M$, то $F(M)$ есть сумма тех элементов матрицы $A$, у которых оба индекса принадлежат $M$. Будем считать, что $F(\varnothing)=0$.

Средним значением функционала $F$ на множестве $I$ будем называть величину

$$
E_{F}(A)=\frac{1}{2^{r}} \sum_{M \subseteq I} F(M) .
$$


Средним значением функщионала $F$ на множестве $I$ для $m$-элементных подмножеств будем называть величину

$$
E_{F, m}(A)=\left(\begin{array}{l}
r \\
m
\end{array}\right)^{-1} \sum_{M \subseteq I,|M|=m} F(M) .
$$

Эти средние величины можно рассматривать как математические ожидания случайных величин, определенных на соответствующих вероятностных пространствах. Для среднего значения функционала $F$ на множестве $I$ элементарными событиями являются все $2^{r}$ подмножеств множества $I$, каждому из них приписывается вероятность $p=2^{-r}$. Для среднего значения функционала $F$ на множестве $I$ для $m$-элементных подмножеств элементарными событиями являются все $\left(\begin{array}{c}r \\ m\end{array}\right) m$-элементных подмножеств множества $I$, и каждому из них приписывается вероятность $p=\left(\begin{array}{c}r \\ m\end{array}\right)^{-1}$.

Через $s(A)$ будем обозначать сумму диагональных элементов матрицы $A$ (след матрицы), а через $v(A)$ - сумму элементов матрицы $A$, лежащих вне диагонали.

Следующая лемма связывает среднее значение функционала $F$ на множестве $I$ с величинами $s(A)$ и $v(A)$.

Лемма 1. Справедливо равенство

$$
E_{F}(A)=\frac{s(A)}{2}+\frac{v(A)}{4}
$$

Доказательство. Рассмотрим сумму значений функционала $F$ по всем подмножествам множества $I$. Диагональный элемент $a_{i i}$ присутствует в этой сумме ровно $2^{r-1}$ раз, так как именно столько подмножеств содержат элемент $i$. Элемент, лежащий вне диагонали, присутствует в этой сумме $2^{r-2}$ раза, так как число подмножеств, содержащих различные элементы $i$ и $j$, равно $2^{r-2}$. Таким образом,

$$
\sum_{M \subseteq I} F(M)=2^{r-1} s(A)+2^{r-2} v(A) .
$$

Поделив полученное равенство на $2^{r}$, получим доказываемое равенство.

Аналогично, используя величины $s(A)$ и $v(A)$, можно получить среднее значение функционала $F$ на множестве $I$ для $m$-элементных подмножеств. Для убывающего факториала будем использовать обозначение

$$
[k]_{l}=k(k-1) \ldots(k-l+1) .
$$

Верна следующая лемма.

Лемма 2. Справедливо равенство

$$
E_{F, m}(A)=\frac{m s(A)}{r}+\frac{[m]_{2} v(A)}{[r]_{2}} .
$$

Доказательство. Посчитаем число вхождений каждого элемента матрицы $A$ в сумму значений функционала $F$ по всем $m$-элементным подмножествам множества $I$. Любой диагональный элемент $a_{i i}$ присутствует в этой сумме ровно $\left(\begin{array}{c}r-1 \\ m-1\end{array}\right)$ раз, так как именно 
столько $m$-элементных подмножеств содержат элемент $i$. Элемент, лежащий вне диагонали, присутствует в этой сумме $\left(\begin{array}{c}r-2 \\ m-2\end{array}\right)$ раза, так как число $m$-элементных подмножеств, содержащих различные элементы $i$ и $j$, равно $\left(\begin{array}{c}r-2 \\ m-2\end{array}\right)$. Таким образом,

$$
\sum_{M \subseteq I,|M|=m} F(M)=\left(\begin{array}{c}
r-1 \\
m-1
\end{array}\right) s(A)+\left(\begin{array}{c}
r-2 \\
m-2
\end{array}\right) v(A) .
$$

Поделив полученное равенство на $\left(\begin{array}{l}r \\ m\end{array}\right)$, получим нужное равенство.

Сопоставим каждому подмножеству $M \subseteq I$ квадрат суммы значений функции $\varphi$ на упорядоченных парах элементов этого подмножества, то есть $F^{2}(M)$. Вычислим значения величин

$$
\begin{aligned}
E_{F^{2}}(A) & =\frac{1}{2^{r}} \sum_{M \subseteq I} F^{2}(M), \\
E_{F^{2}, m}(A) & =\left(\begin{array}{c}
r \\
m
\end{array}\right)^{-1} \sum_{M \subseteq I,|M|=m} F^{2}(M) .
\end{aligned}
$$

Поскольку значения функционала $F(M)$ для всех подмножеств и подмножеств фиксированной мощности рассматриваются как случайные величины, значения $E_{F^{2}}(A)$ и $E_{F^{2}, m}(A)$ являются, соответственно, их вторыми моментами.

Введем обозначения для ряда величин, определяемых по матрице $A$, которые будут использованы в дальнейшем. Элементы матрицы $a_{i i}, i=0,1, \ldots, 2^{n}-1$, будем называть диагональными, а остальные - недиагональными. Пару элементов матрицы $a_{i j}$ и $a_{j, i}$, $i \neq j$, будем называть симметричной относительно диагонали, а остальные пары несимметричными. Строки и столбцы будем называть линиями. Если элементы лежат в одной линии, то они лежат либо в одной строке, либо в одном столбце. Если один из элементов матрицы лежит в некоторой строке, а другой лежит в столбце, пересекающемся с данной строкой по диагональному элементу, то будем говорить, что элементы лежат в различных линиях с одним номером.

Пусть

$q_{1}(A)$ - сумма квадратов диагональных элементов матрицы $A$;

$q_{2}(A)$ - сумма квадратов недиагональных элементов матрицы $A$;

$p_{1}(A)$ - сумма произведений неупорядоченных пар, симметричных относительно диагонали элементов матрицы $A$;

$p_{2}(A)$ - сумма произведений неупорядоченных пар различных (по индексам) элементов матрищы $A$, один из которых диагональный, а другой либо тоже диагональный, либо лежит в одной линии с первым диагональным элементом;

$p_{3}(A)$ - сумма произведений неупорядоченных пар различных недиагональных элементов матрицы $A$, лежащих в одной линии;

$p_{4}(A)$ - сумма произведений неупорядоченных пар различных недиагональных несимметричных элементов матрицы $A$, лежащих в линиях с одним номером; 
$p_{5}(A)$ - сумма произведений неупорядоченных пар различных элементов матрицы $A$, один из которых диагональный, а другой недиагональный и не лежит с диагональным элементом в одной линии;

$p_{6}(A)$ - сумма произведений неупорядоченных пар различных недиагональных несимметричных элементов матрицы $A$, не лежащих в одной линии или в линиях с одним номером.

Заметим, что в $p_{1}(A)$ и $p_{2}(A)$ входят пары элементов матрицы, объединение множеств индексов которых содержит ровно два различных индекса, в $p_{3}(A), p_{4}(A)$ и $p_{5}(A)-$ соответственно ровно три различных индекса, наконец в $p_{6}(A)$ - четыре различных индекса. Можно было группировать их именно по этому признаку, но выделение пар с диагональными элементами упрощает вычисления при условии равенства нулю диагональных элементов. Величины $q_{1}(A), p_{2}(A)$ и $p_{5}(A)$ при этом просто равны нулю.

Следующее утверждение дает выражение величины $E_{F^{2}}(A)$ через выше введенные величины.

Лемма 3. Справедливо равенство

$$
\begin{aligned}
E_{F^{2}}(A)=\frac{4 q_{1}(A)+2 q_{2}(A)+4 p_{1}(A)+4 p_{2}(A)}{8} & \\
+ & \frac{2 p_{3}(A)+2 p_{4}(A)+2 p_{5}(A)+p_{6}(A)}{8} .
\end{aligned}
$$

Доказательство. Рассмотрим величину $\sum_{M \subseteq I} F^{2}(M)$ как сумму квадратов сумм элементов соответствующих подматриц, строки и столбцы которых задаются подмножествами $M \subseteq I$. Посчитаем вклад каждого элемента и каждой неупорядоченной пары элементов в величину $\sum_{M \subseteq I} F^{2}(M)$. Диагональный элемент $a_{i i}$ входит ровно в $2^{r-1}$ подматриц, определяемых подмножествами индексов $M$, следовательно, его вклад будет равен $2^{r-1} a_{i i}^{2}$. Недиагональный элемент $a_{i j}, i \neq j$, входит в $2^{r-2}$ подматриц, и его вклад будет равен $2^{r-2} a_{i j}^{2}$. Неупорядоченная пара симметричных элементов содержится в $2^{r-2}$ подматрицах. Неупорядоченная пара различных диагональных элементов содержится в $2^{r-2}$ подматрицах. Пара из диагонального и любого другого, лежащего с ним в одной линии, тоже входит ровно в $2^{r-2}$ подматриц. Если пара состоит из двух различных элементов, находящихся в одной линии и не лежащих на диагонали, то существует ровно $2^{r-3}$ содержащих их подматрищ. Столько же существует подматриц, содержащих данную неупорядоченную пару недиагональных несимметричных элементов, лежащих в линиях с одним номером. Столько же существует подматриц, содержащих фиксированный диагональный элемент и не находящийся с ним в одной линии недиагональный элемент. Наконец, неупорядоченная пара недиагональных элементов, не лежащих в одной линии или в линиях с одним номером, входит ровно в $2^{r-4}$ подматриц. Следовательно, имеет место равенство

$$
\begin{aligned}
\sum_{M \subseteq I} F^{2}(M)=2^{r-1} q_{1}(A) & +2^{r-2} q_{2}(A)+2 \cdot 2^{r-2} p_{1}(A)+2 \cdot 2^{r-2} p_{2}(A) \\
& +2 \cdot 2^{r-3} p_{3}(A)+2 \cdot 2^{r-3} p_{4}(A)+2 \cdot 2^{r-3} p_{5}(A)+2 \cdot 2^{r-4} p_{6}(A) .
\end{aligned}
$$

Из этого равенства непосредственно следует утверждение леммы.

Сформулируем и докажем аналогичное утверждение для величины $E_{F^{2}, m}(A)$. 
Лемма 4. Справедливо равенство

$$
\begin{aligned}
& E_{F^{2}, m}(A)=\frac{m q_{1}(A)}{r}+\frac{[m]_{2}\left(q_{2}(A)+2 p_{1}(A)+2 p_{2}(A)\right)}{[r]_{2}} \\
&+\frac{[m]_{3}\left(2 p_{3}(A)+2 p_{4}(A)+2 p_{5}(A)\right)}{[r]_{3}}+\frac{[m]_{4} 2 p_{6}(A)}{[r]_{4}}
\end{aligned}
$$

Доказательство. Аналогично доказательству предыдущей леммы посчитаем вклад каждого элемента и каждой неупорядоченной пары элементов матрицы в сумму $\sum_{M \subseteq I,|M|=m} F^{2}(M)$. Как и в предыдущей лемме, индексы выбранных элементов или неупорядоченных пар элементов фиксируют строки и столбцы, номера которых совпадают с одним из индексов выбранных элементов. Номера строк и столбцов, дополняющих выбранные до нужного числа $m$, выбираются произвольно из оставшегося множества индексов. Получим, что

$$
\begin{aligned}
\sum_{M \subseteq I,|M|=m} F^{2}(M)= & \left(\begin{array}{c}
r-1 \\
m-1
\end{array}\right) q_{1}(A)+\left(\begin{array}{c}
r-2 \\
m-2
\end{array}\right)\left(q_{2}(A)+2 p_{1}(A)+2 p_{2}(A)\right) \\
& +\left(\begin{array}{c}
r-3 \\
m-3
\end{array}\right)\left(2 p_{3}(A)+2 p_{4}(A)+2 p_{5}(A)\right)+\left(\begin{array}{c}
r-4 \\
m-4
\end{array}\right) 2 p_{6}(A) .
\end{aligned}
$$

Лемма непосредственно следует из этого равенства.

Введем, наконец, обозначения для следующих величин. Пусть

$$
\begin{aligned}
D_{F}(A) & =E_{F^{2}}(A)-E_{F}^{2}(A), \\
D_{F, m}(A) & =E_{F^{2}, m}(A)-E_{F, m}^{2}(A) .
\end{aligned}
$$

Ясно, что для случайных величин, определенных для всех подмножеств или подмножеств фиксированной мощности, значения величин $D_{F}(A)$ и $D_{F, m}(A)$ являются соответственно их дисперсиями, а $\sqrt{D_{F}(A)}$ и $\sqrt{D_{F, m}(A)}-$ их стандартными отклонениями.

Заметим, что общий характер полученных формул позволяет применять их для вычисления средних и типичных значений для произвольных функционалов, определенных на подмножествах конечного множества как сумма значений, приписываемых упорядоченным парам элементов этого множества.

\section{3. Средние значения сумм попарных расстояний}

Применим доказанные вспомогательные утверждения для нахождения средних и оценки типичных значений для функщионала, являющегося суммой попарных расстояний между неупорядоченными парами вершин множества $M$ мощности $m$, являющегося подмножеством вершин $n$-мерного единичного куба.

Пусть $\tilde{\alpha}_{i}^{n}$ - вершина $n$-мерного единичного куба с лексикографическим номером $i$. Зададим матрицу $A$ порядка $2^{n}$ следующим образом. Элемент $a_{i j}$ равен расстоянию Хэмминга $\rho\left(\tilde{\alpha}_{i}^{n}, \tilde{\alpha}_{j}^{n}\right)$ между вершинами $\tilde{\alpha}_{i}^{n}$ и $\tilde{\alpha}_{j}^{n}, i, j=0,1, \ldots, 2^{n}-1$, нумерация срок и столбцов начинается с 0. Отметим сразу, что эта матрица симметричная и на ее диагонали стоят нули. Любые строка или столбец являются перестановками элементов любых 
других строки или столбца. Сумма элементов матрицы в любой строке и в любом столбце этой матрицы равна одной и той же величине. Найдем ее (см. [5], стр. 264):

$$
\sum_{j=0}^{2^{n}-1} a_{i j}=\sum_{i=0}^{2^{n}-1} a_{i j}=\sum_{k=0}^{n} k\left(\begin{array}{l}
n \\
k
\end{array}\right)=n 2^{n-1} .
$$

Введенные выше величины $s(A)$ и $v(A)$ имеют следующие значения:

$$
s(A)=0, \quad v(A)=n 2^{2 n-1} .
$$

Пусть $M \subseteq B^{n}-$ произвольное множество, состоящее из различных вершин $n$-мерного единичного куба. Сопоставим этому множеству величину

$$
R(M)=\frac{1}{2} \sum_{\tilde{\alpha}^{n} \in M, \tilde{\beta}^{n} \in M} \rho\left(\tilde{\alpha}_{n}, \tilde{\beta}_{n}\right)
$$

- функционал суммы попарных расстояний. Если множество $M$ пусто или состоит только из одной вершины, то будем считать, что $R(M)=0$. Ясно, что величина $\sum_{\tilde{\alpha}^{n} \in M, \tilde{\beta}^{n} \in M} \rho\left(\tilde{\alpha}_{n}, \tilde{\beta}_{n}\right)$ равна сумме элементов матрицы $A$, индексы которых совпадают с лексикографическими номерами вершин из множества $M$. Для перехода от упорядоченных пар к неупорядоченным достаточно уменьшить эту сумму в два раза. Это следует из симметричности матрицы и равенства нулю ее диагональных элементов.

Средним значением суммы попарных расстояний для всех подмножеств $B^{n}$ будем называть величину

$$
S(n)=\frac{1}{2^{2^{n}}} \sum_{M \subseteq B^{n}} R(M)
$$

Непосредственно из леммы 1 вытекает следующее утверждение.

Теорема 1. Справедливо равенство

$$
S(n)=n 4^{n-2}
$$

Средним значением суммы попарных расстояний для подмножеств $B^{n}$ мощности $m$ будем называть величину

$$
S(n, m)=\left(\begin{array}{l}
2^{n} \\
m
\end{array}\right)^{-1} \sum_{M \subseteq B^{n},|M|=m} R(M) .
$$

Используя лемму 2, получим следующее утверждение.

Теорема 2. Справедливо равенство

$$
S(n, m)=\frac{m^{2} n}{4}\left(1-\frac{1}{m}\right)\left(1+\frac{1}{2^{n}-1}\right) .
$$

Доказательство. Действительно, из леммы 2 и вычисленных значений величин $s(A)$ и $v(A)$ получим, что

$$
S(n, m)=\frac{[m]_{2} 2^{2 n-1}}{\left[2^{n}\right]_{2} 2}=\frac{m^{2} n}{4}\left(1-\frac{1}{m}\right)\left(1+\frac{1}{2^{n}-1}\right) .
$$


Напомним, что наибольшее возможное значение суммы попарных расстояний для $m$-элементных подмножеств вершин $n$-мерного единичного куба $U(n, m)$ равно $n\lfloor m / 2\rfloor\lceil m / 2\rceil$, то есть не превосходит $m^{2} n / 4$ (для четных $m$ эта оценка достигается, см. [6]). Сравнивая это с полученным средним значением $S(n, m)$, получим следующее утверждение.

Теорема 3. Пусть $n, m \rightarrow \infty$, тогда

$$
S(n, m) \sim U(n, m)
$$

Известна также нижняя оценка суммы попарных расстояний для $m$-элементных подмножеств вершин $n$-мерного единичного куба

$$
R(M) \geqslant \frac{m^{2} n}{4}\left(1+\frac{1}{n}-\frac{2^{n}}{m n}\right),
$$

которая достигается для подмножеств мощности $2^{n-1}$, являющихся $(n-1)$-мерными гранями куба $B^{n}$ (см. [6]). Из этого результата вытекает, что если мощность $m$-элементных подмножеств вершин является достаточно быстро растущей функцией, то нижняя и верхняя оценки для суммы попарных расстояний асимптотически совпадают. Сформулируем это утверждение в виде теоремы.

Теорема 4. Пусть

$$
m(n)=\frac{2^{n} \alpha(n)}{n}
$$

где функиия $\alpha(n)$ зависит от натурального $n$, принимает действительные значения $и$ $\alpha(n) \rightarrow \infty$ nрu $n \rightarrow \infty$, тогдa

$$
L(n, m(n)) \sim U(n, m(n)) .
$$

Этому факту можно дать следующую качественную интерпретацию. Если множества являются более плотными в $B^{n}$, чем код Хэмминга, то все они имеют асимптотически одинаковую сумму попарных расстояний.

\section{4. Типичные значения сумм попарных расстояний}

Вычислим теперь для матрицы $A$ попарных расстояний между вершинами $n$-мерного единичного куба значения величин $q_{1}(A), q_{2}(A), p_{1}(A), p_{2}(A), p_{3}(A), p_{4}(A), p_{5}(A)$ и $p_{6}(A)$.

Очевидно, из условия, что на диагонали матрицы $A$ стоят нули, следует, что $q_{1}(A)=0$, $p_{2}(A)=0$ и $p_{5}(A)=0$.

Сумма квадратов элементов матрицы, не лежащих на диагонали, в любой строке и в любом столбце этой матрицы равна одной и той же величине. Найдем ее (см. [5], стр. 264):

$$
\sum_{j=0}^{2^{n}-1} a_{i j}^{2}=\sum_{i=0}^{2^{n}-1} a_{i j}^{2}=\sum_{k=0}^{n} k^{2}\left(\begin{array}{l}
n \\
k
\end{array}\right)=n(n+1) 2^{n-2} .
$$


Отсюда следует, что

$$
q_{2}(A)=\frac{n(n+1) 4^{n}}{4}
$$

Найдем значение $p_{1}(A)$. Матрица $A$ симметрична, поэтому

$$
p_{1}(A)=\sum_{0 \leqslant i<j \leqslant 2^{n}-1} a_{i j} a_{j, i}=\sum_{0 \leqslant i<j \leqslant 2^{n}-1} a_{i j}^{2}=\frac{n(n+1) 4^{n}}{8}=\frac{q_{2}(A)}{2} .
$$

Сумма произведений неупорядоченных пар различных недиагональных элементов матрищы $A$, лежащих в одной фиксированной линии, равна

$$
\begin{aligned}
\frac{1}{2}\left(\left(\sum_{j=0}^{2^{n}-1} a_{i j}\right)^{2}-\sum_{j=0}^{2^{n}-1} a_{i j}^{2}\right) & =\frac{1}{2}\left(\left(\sum_{i=0}^{2^{n}-1} a_{i j}\right)^{2}-\sum_{j=0}^{2^{n}-1} a_{i j}^{2}\right) \\
& =\frac{n^{2} 4^{n}-n(n+1) 2^{n}}{8} .
\end{aligned}
$$

Общее число строк и столбцов матрицы равно $2^{n+1}$, поэтому

$$
p_{3}(A)=\frac{n^{2} 8^{n}-n(n+1) 4^{n}}{4} .
$$

Найдем значение $p_{4}(A)$ :

$$
\begin{aligned}
p_{4}(A) & =\frac{1}{2} \sum_{i=0}^{2^{n}-1} \sum_{j=0}^{2^{n}-1} a_{i j}\left(2 n 2^{n-1}-2 a_{j, i}\right) \\
& =n 2^{n-1} \sum_{i=0}^{2^{n}-1} \sum_{j=0}^{2^{n}-1} a_{i j}-\sum_{i=0}^{2^{n}-1} \sum_{i=0}^{2^{n}-1} a_{i j}^{2}=\frac{n^{2} 8^{n}-n(n+1) 4^{n}}{4}=p_{3}(A) .
\end{aligned}
$$

Найдем, наконец, значение величины $p_{6}(A)$. Для этого вычислим сумму произведений каждого элемента матрищы на сумму всех оставшихся элементов, не лежащих с ним в одной линии и в линиях с одинаковыми номерами, и поделим ее на 2 . В результате получим, что

$$
\begin{aligned}
p_{6}(A) & =\frac{1}{2} \sum_{i=0}^{2^{n}-1} \sum_{j=0}^{2^{n}-1} a_{i j}\left(n 2^{n-1} 2^{n}-4 n 2^{n-1}+a_{i j}+a_{j, i}\right) \\
& =\left(\frac{n 4^{n}}{4}-n 2^{n}\right) \sum_{i=0}^{2^{n}-1} \sum_{j=0}^{2^{n}-1} a_{i j}+\sum_{i=0}^{2^{n}-1} \sum_{j=0}^{2^{n}-1} a_{i j}^{2} \\
& =\frac{n^{2} 16^{n}-4 n^{2} 8^{n}+2 n(n+1) 4^{n}}{8} .
\end{aligned}
$$

Средним значением квадрата суммы попарных расстояний для всех подмножеств $B^{n}$ будем называть величину

$$
Q(n)=\frac{1}{2^{2^{n}}} \sum_{M \subseteq B^{n}} R^{2}(M)
$$


Найдем величину $Q(n)$. Используем лемму 3 , заметим, что для перехода от упорядоченных пар вершин к неупорядоченыым достаточно уменьшить эту сумму в 4 раза. Поэтому

$$
\begin{aligned}
Q(n) & =\frac{2 q_{2}(A)+4 p_{1}(A)+2 p_{3}(A)+2 p_{4}(A)+p_{6}(A)}{32} \\
& =\frac{n^{2} 16^{n}+4 n^{2} 8^{n}+2 n^{2} 4^{n}+2 n 4^{n}}{256} .
\end{aligned}
$$

Пусть $D(n)=Q(n)-S^{2}(n)$, тогда

$$
D(n)=\frac{2 n^{2} 8^{n}+n^{2} 4^{n}+n 4^{n}}{128} .
$$

Теорема 5. Пусть функиия $\alpha(n)$ зависит от натурального $n$, принимает действительные значения $и \alpha(n) \rightarrow \infty$ при $n \rightarrow \infty$. Тогда для почти всех подмножеств $M \subseteq B^{n}$ имеет место неравенство

$$
|R(M)-S(n)|<\frac{\alpha(n) S(n)}{\sqrt{2^{n}}} .
$$

Доказательство. Будем рассматривать $R(M)$ как случайную величину, определенную на вероятностном пространстве, элементарными событиями которого являются все $2^{2^{n}}$ подмножеств множества $B^{n}$, каждому из них приписывается вероятность $p=1 / 2^{2^{n}}$, $S(n)$ и $D(n)$ являются соответственно математическим ожиданием и дисперсией этой случайной величины. Полагая в неравенстве Чебышева

$$
\mathbf{P}\{|R(M)-S(n)| \geqslant t\} \leqslant \frac{D(n)}{t^{2}}
$$

величину $t$ равной $t(n)=\alpha(n) S(n) / \sqrt{2^{n}}$ и учитывая, что

$$
\frac{D(n)}{S^{2}(n)}=\frac{4 n^{2} 8^{n}+2 n^{2} 4^{n}+2 n 4^{n}}{n^{2} 16^{n}}=\frac{4}{2^{n}}+\frac{2}{4^{n}}+\frac{2}{n 4^{n}},
$$

получим, что $D(n) / t^{2} \rightarrow 0$ при $n \rightarrow \infty$, откуда и следует утверждение теоремы.

Средним значением квадрата суммы попарных расстояний для подмножеств $B^{n}$ мощности $m$ будем называть величину

$$
Q(n, m)=\left(\begin{array}{l}
2^{n} \\
m
\end{array}\right)^{-1} \sum_{M \subseteq B^{n},|M|=m} R^{2}(M) .
$$

Найдем величину $Q(n, m)$. Используем лемму 4 , уменьшим эту сумму в 4 раза для того, чтобы перейти от упорядоченных пар вершин к неупорядоченным. В результате получим, что

$$
\begin{aligned}
Q(n, m)= & \frac{1}{4}\left(\frac{[m]_{2}\left(q_{2}(A)+2 p_{1}(A)\right)}{\left[2^{n}\right]_{2}}+\frac{[m]_{3}\left(2 p_{3}(A)+2 p_{4}(A)\right)}{\left[2^{n}\right]_{3}}+\frac{[m]_{4} 2 p_{6}(A)}{\left[2^{n}\right]_{4}}\right) \\
= & \frac{[m]_{2}}{\left[2^{n}\right]_{2}} \frac{n(n+1) 4^{n}}{8}+\frac{[m]_{3}}{\left[2^{n}\right]_{3}} \frac{n^{2} 8^{n}-n(n+1) 4^{n}}{4} \\
& +\frac{[m]_{4}}{\left[2^{n}\right]_{4}} \frac{n^{2} 16^{n}-4 n^{2} 8^{n}+2 n(n+1) 4^{n}}{16}
\end{aligned}
$$


Пусть $D(n, m)=Q(n, m)-S^{2}(n, m)$, тогда

$$
\begin{aligned}
D(n, m)=\frac{[m]_{2}}{\left[2^{n}\right]_{2}} \frac{n(n+1) 4^{n}}{8} & +\frac{[m]_{3}}{\left[2^{n}\right]_{3}} \frac{n^{2} 8^{n}-n(n+1) 4^{n}}{4} \\
& +\frac{[m]_{4}}{\left[2^{n}\right]_{4}} \frac{n^{2} 16^{n}-4 n^{2} 8^{n}+2 n(n+1) 4^{n}}{16}-\left(\frac{[m]_{2}}{\left[2^{n}\right]_{2}}\right)^{2} \frac{n^{2} 16^{n}}{16}
\end{aligned}
$$

Рассмотрим частное $D(n, m) / S^{2}(n, m)$. Ясно, что

$$
\begin{aligned}
\frac{D(n, m)}{S^{2}(n, m)}= & \frac{2}{m(m-1)}\left(1-\frac{1}{2^{n}}\right)\left(1+\frac{1}{n}\right) \\
& +\frac{4}{m}\left(1-\frac{1}{m-1}\right)\left(1+\frac{1}{2^{n}-2}\right)\left(1-\frac{1}{2^{n}}-\frac{1}{n 2^{n}}\right) \\
& +\left(1-\frac{2}{m}\right)\left(1-\frac{2}{m-1}\right)\left(1+\frac{2}{2^{n}-2}\right) \\
& \times\left(1+\frac{2}{2^{n}-3}\right)\left(1-\frac{4}{2^{n}}+\frac{2}{4^{n}}+\frac{2}{n 4^{n}}\right)-1 .
\end{aligned}
$$

Заметим, что

$$
\frac{2}{m(m-1)}=2 \sum_{k=2}^{\infty} \frac{1}{m^{k}}, \quad \frac{4}{m}\left(1-\frac{1}{m-1}\right)=\frac{4}{m}-4 \sum_{k=2}^{\infty} \frac{1}{m^{k}}
$$

и

$$
\left(1-\frac{2}{m}\right)\left(1-\frac{2}{m-1}\right)=1-\frac{4}{m}+2 \sum_{k=2}^{\infty} \frac{1}{m^{k}}
$$

Преобразуем произведения в ряды:

$$
\left(1+\frac{1}{2^{n}-2}\right)\left(1-\frac{1}{2^{n}}-\frac{1}{n 2^{n}}\right)=1+\sum_{k=2}^{\infty} \frac{2^{k-2}}{\left(2^{n}\right)^{k}}-\frac{1}{n 2^{n}}\left(1+\sum_{k=1}^{\infty} \frac{2^{k-1}}{\left(2^{n}\right)^{k}}\right)
$$

и

$$
\begin{aligned}
\left(1+\frac{2}{2^{n}-2}\right)\left(1+\frac{2}{2^{n}-3}\right) & \left(1-\frac{4}{2^{n}}+\frac{2}{4^{n}}+\frac{2}{n 4^{n}}\right) \\
& =1-\sum_{k=3}^{\infty} \frac{2 \cdot 3^{k-2}-2^{k-1}}{\left(2^{n}\right)^{k}}+\frac{2}{n 4^{n}}\left(1+\sum_{k=1}^{\infty} \frac{2 \cdot 3^{k}-2^{k}}{\left(2^{n}\right)^{k}}\right) .
\end{aligned}
$$

Приведем подобные члены, произведем другие несложные преобразования, учтем, что $m \leqslant 2^{n}$, и получим неравенство

$$
\frac{D(n, m)}{S^{2}(n, m)} \leqslant \frac{4}{m^{2} n}(1+O(1 / m))
$$


Теорема 6. Пусть функция $\alpha(n)$ зависит от натурального $n$, принимает действительные значения $и \alpha(n) \rightarrow \infty$ при $n \rightarrow \infty$. Тогда для почти всех подмножеств $M \subseteq B^{n}$ таких, что $|M|=m$, где $m=m(n) \rightarrow \infty$ при $n \rightarrow \infty$, имеет место неравенство

$$
|R(M)-S(n, m)|<\frac{\alpha(n) S(n, m)}{m \sqrt{n}}
$$

Доказательство. Будем рассматривать $R(M)$ как случайную величину, определенную на вероятностном пространстве, элементарными событиями которого являются все $\left(\begin{array}{l}2^{n} \\ m\end{array}\right)$ подмножеств множества $B^{n}$, каждому из них приписывается вероятность $p=\left(\begin{array}{c}2_{m}^{n} \\ m\end{array}\right)^{-1}$, $S(n, m)$ и $D(n, m)$ являются соответственно математическим ожиданием и дисперсией этой случайной величины. Полагая в неравенстве Чебышева

$$
\mathbf{P}\{|R(M)-S(n, m)| \geqslant t\} \leqslant \frac{D(n, m)}{t^{2}}
$$

величину $t$ равной $t(n)=\alpha(n) S(n, m)(m \sqrt{n})$ и учитывая, что

$$
\frac{D(n, m)}{S^{2}(n, m)} \leqslant \frac{4}{m^{2} n}(1+O(1 / m))
$$

получим, что $D(n, m) / t^{2} \rightarrow 0$ при $n \rightarrow \infty$, откуда следует утверждение теоремы.

\section{Список литературы}

1. Зильберман Б. С., О расположении зарядов в вершинах единичного $n$-мерного куба. Докл. $A H$ СССР (1963) 149, №3, 546-549.

2. Круглова Т. Н., Об асимптотическом методе решения задачи о зарядах. Проблемы кибернетики (1965) 13, 29-44.

3. Леонтьев В. К., Асимптотически устойчивые расположения зарядов в вершинах единичного n-мерного куба. Проблемы кибернетики (1970) 23, 29-42.

4. Перина Е. В., О множествах вершин $n$-мерного единичного куба с максимальной энергией. Проблемы кибернетики (1974) 27, 279-292.

5. Гаврилов Г. П., Сапоженко А. А., Задачи и упражнения по курсу дискретной математики. Наука, Москва, 1992.

6. Воронин В. П., О множествах вершин $N$-мерного единичного куба с минимальной суммой попарных расстояний. В кн.: Вопросы кибернетики. Комбинаторный анализ и теория графов. Изд-во Научного совета по комплексной проблеме "Кибернетика" АН СССР, Москва, 1980, c. 48-57.

Статья поступила 10.11.2003. 